\title{
Physical properties of palm based Diacylglycerol and palm-based oils in the preparation of shelf-stable margarine
}

\begin{abstract}
Ternary mixtures containing palm olein (POL), palm kernel oil (PKO) and palm oil-based diacylglycerol (PO-DAG) were designed using mixture design. The corresponding physical properties such as solid fat content (SFC) as well as deviation from SFC ( $\triangle \mathrm{SFC})$ using nuclear magnetic resonance (NMR) and melting and crystallization properties using differential scanning calorimetry (DSC) were studied. Ternary phase behaviour was analysed using isosolid diagrams. The most intensive eutectic interaction among the three binary blends studied was observed along the binary line of PKO/PO-DAG followed by POL/PKO and POL/PO-DAG. The higher $\triangle \mathrm{SFC}$ did not always lead to the more intensive eutectic behaviour among the blends. Addition of pure POL, 33.33 and $66.66 \%$ POL, and no POL to $50 / 50$ mixture of $\mathrm{PKO} / \mathrm{PO}-\mathrm{DAG}$ decreased heat of crystallization $(\Delta \mathrm{Hc})$ as well as crystallization onset (TO). However, as the same amounts of PO-DAG and PKO were added to the 50/50 mixtures of POL/PKO and POL/PO-DAG, respectively, blend containing the equi-mixture of POL, PKO and PO-DAG (33.33/33.33/33.33) was found to have the lowest $\Delta$ Hc. This was also reflected in the corresponding eutectic effect observed at 20-25 and 5$10^{\circ} \mathrm{C}$, respectively. Palm-based DAG-enriched shelf-stable margarine consisting of POL/PKO/PO-DAG (42.5/42.5/15 w/w) was optimally formulated through analysis of multiple isosolid diagrams and was found to have quite similar SFC profile with commercial shelf-stable margarine.
\end{abstract}

Keyword: Design-expert; Isosolid diagram; Palm oil-based diacylglycerol; Shelf-stable margarine; Ternary blend 